\title{
Molecular cloning and characterization of the actin-depolymerizing factor gene in Gossypium barbadense
}

\author{
Jina $\mathrm{Chi}^{1}$, Xingfen Wang ${ }^{1}$, Hongmei Zhou ${ }^{1}$, Guiyin Zhang ${ }^{1}$, \\ Yanxiang Sun ${ }^{2}$, Zhikun $\mathrm{Li}^{1}$ and Zhiying $\mathrm{Ma}^{1 *}$ \\ ${ }^{1}$ Key Laboratory of Crop Germplasm Resources of Hebei Province, Agricultural \\ University of Hebei, Baoding, 071001, Hebei, P. R. China \\ ${ }^{2}$ College of Life Science, Normal University of Langfang, Langfang, \\ 102800, Hebei, P. R. China
}

(Received 25 April 2008, accepted 2 September 2008)

\begin{abstract}
The Sea Island cotton (Gossypium barbadense L.) has been highly valued in verticillium wilt resistance and many fiber qualities including fiber length, strength and fineness. To identify whether it had some special genes in fiber development in comparison with the Upland cotton (G. hirsutum L.), an actin-depolymerizing factor (ADF) gene was cloned and characterized in this research. A $420 \mathrm{bp}$ open reading frame of the cloned gene, named $G b A D F 1$, encoded a protein of 139 amino acids, including $39.57 \%$ nonpolar amino acids, $17.27 \%$ acidic amino acids, $15.83 \%$ basic amino acids and $31.92 \%$ hydrophobic amino acids. Its molecular weight was about $15 \mathrm{kDa}$, and $\mathrm{pI} 5.04$. GbADF1 contained two conserved domains, 6-Ser and the PIP2/actin binding site. Its amino acid sequence was similar to the ADF/ cofilin family of other plants. Compared with cDNA sequence, the GbADF1 gene contained one intron near the 3' end in genomic sequence. Semi-quantitative RTPCR result showed that $G b A D F 1$ was a constitutive expression gene in cotton, and higher expression level was detected in fibers than in trophic tissues. The GbADF1 was successfully expressed as a fusion protein in Escherichia coli BL21 (DE3). The molecular weight was firstly calculated by SDS-PAGE. Western blotting analysis confirmed the existence of a protein corresponding to GbADF1. The structure of $G b A D F 1$ was different from that of other ADF genes in higher plant, although the coding sequences of all cloned $A D F s$ were highly conserved.
\end{abstract}

Key words: ADF (actin-depolymerizing factor), semi-quantitative RT-PCR, Sea Island cotton, western blotting

\section{INTRODUCTION}

The plant actin cytoskeleton is known to play key roles in morphogenesis and function of highly specialized cell types, such as root hairs, trichomes (leaf hairs), pollen tubes and stomatal guard cells. Multiple actin binding proteins, such as actin-depolymerizing factors (ADF)/ cofilins, profilins etc., regulate actin dynamics by maintaining the optimum equilibrium between unpolymerized actin molecules (G-actin) and assembled actin filaments (F-actin) in different cellular processes (Hussey et al., 2006). ADF/cofilin family, highly conserved in eukaryotic cells, is a large ubiquitous family with low molecular

Edited by Masao Tasaka

* Corresponding author. E-mail: mzhy@hebau.edu.cn

Note: Nucelotide sequence data reported is available in the GenBank database under the accession no. EU165514. mass (15-22 kDa) (Staiger et al., 1997). In eukaryotic cells, the ADF/cofilin family is an essential group of actin regulatory proteins that enhance the turnover of G-actin and $\mathrm{F}$-actin by regulating the rate constants of dual functions, not only promoting the depolymerization, but also accelerating the polymerization of F-actin under certain conditions (Lappalainen and Drubin, 1997; Bamburg et al., 1999; Gibbon, 2001). ADF is more potent in depolymerization than cofilin (Giuliano et al., 1988).

In animals and yeast systems, genes encoding $\mathrm{ADF} /$ cofilins have been cloned and identified from a variety of organisms (Iida et al., 1993; Moon et al., 1993; Aizawa et al., 1995; Gunsalus et al., 1995). In higher plant, cDNAs encoding ADFs have been cloned and characterized from Lilium longiflorum (Kim et al., 1993), Brassica napus (Kim et al., 1993), Zea mays (Kim et al., 1993; Rozycka et al., 1995; Lopez et al., 1996; Hussey et al., 1998), 
Arabidopsis (Dong et al., 2001a; 2001b) and G. hirsutum (Zhang et al., 2006, 2007). Overexpression of AtADF1 caused irregular tissue morphogenesis and reduced the growth of cells and organs in Arabidopsis (Dong et al., 2001a). Overexpression of AtADF1, AtADF5 and $A t A D F 6$ in different types of plant cells reorganized the actin cytoskeleton and reduced the length and number of filamentous actin structures (Dong et al., 2001b). The heterogenetic expression of $A t A D F 4$ gene in tobacco resulted in wavied hypocotyls of transgenic plants, especially in darkness (Peng and Huang, 2006). In contrast, reduced $A t A D F s$ expression promoted the formation of actin cables, stimulated cell expansion as well as organ growth, and resulted in a delay in Arabidopsis flowering (Dong et al., 2001a).

Although ADFs in Arabidopsis had been well characterized, little such research was done about cotton $A D F s$. In cotton, eight $G h A D F s$ with completed coding sequences could be found in NCBI (the National Center for Biotechnology Information). GhADF1 shared $100 \%$ identity with $G h A D F 8$ in amino acids, but there were only sequences in NCBI and no more other information. Four of GhADFs were isolated from cotton fiber cDNA library and analyzed by the molecular evolutionary relationship (Zhang et al., 2007). GhADF2 and GhADF3 all encoded 139 amino acids, and shared 99\% identity with each other. GhADF4 deduced a protein with 143 amino acids and was closely related to AtADF6. GhADF5 encoded a protein of 141 amino acids and was closely related to AtADF5. RT-PCR analysis showed that GhADF2 was predominantly expressed in fiber, GhADF3 and GhADF4 in each tissue, and GhADF5 mainly in cotyledons. Expression levels of different $G h A D F s$ showed a discrepancy in the same cell type (Zhang et al., 2007), suggesting that they might be involved in regulating the development of cotton fiber.

In this study, we want to identify and characterize the $G b A D F$ involved in fiber development of $G$. barbadense. As far as we are aware, this work represents the first published $A D F s$ study of Sea Island cotton.

\section{MATERIALS AND METHODS}

Cotton materials The Sea Island cotton (cv. Pima 9053) was planted in a field. Developing bolls were tagged from the starting day post anthesis (DPA) on. All developing fibers of 10-20 DPA were removed from the seeds of the tagged bolls. Cotyledons, hypocotyls and roots were collected from two-week-old young seedlings. The fibers and the collected tissues were immediately frozen in liquid nitrogen for extraction of nucleic acids.

Isolation and purification of cotton DNA and total RNA The genomic DNA (gDNA) of Pima90-53 was extracted from plantlets with two cotyledons according to the published protocol (Paterson et al., 1993). Total RNA was extracted from fibers of 10-20 DPA, roots, cotyledons and hypocotyls with PlantRNA reagent (Tiangen, China) according to the manufacture's instruction, respectively. The isolated RNA was treated by RNase-free DNase I according to manufacture's protocol to remove any potential gDNA contamination. The purity and concentration of the total RNA were determined using gel electrophoresis and Beckman spectrophotometer.

Isolation of the full-length coding sequence encoding $\boldsymbol{G} \boldsymbol{b} \boldsymbol{A} \boldsymbol{D} \boldsymbol{F}$ from cotton Bioinformatics methods and the RT-PCR procedure were combined to clone the fulllength open reading frame (ORF) of GbADF1 from Pima90-53. First, an EST fragment of 511 bp homologous to $A D F$ gene was identified by sequencing $42 \mathrm{PCR}$ products from cotton fiber. Four cotton ESTs, DW500081, DW482538, DW482537 and DR459201, were found in NCBI. A 729 bp sequence was assembled via in silico cloning. According to the sequence, full length ORF was amplified and sequenced. The primers PF: 5'-ATGGCAAACGCGGCTTCAG-3' and PR: 5'-TCAATTGGCACGGCTCCTTATG-3' were designed. Then the PCR was run as follows: one cycle of $1 \mathrm{~min}$ at $95^{\circ} \mathrm{C}, 30$ cycles of 1 min at $94^{\circ} \mathrm{C}, 1 \mathrm{~min}$ at $58^{\circ} \mathrm{C}, 1.5 \mathrm{~min}$ at $72^{\circ} \mathrm{C}$, and a final extension of $10 \mathrm{~min}$ at $72^{\circ} \mathrm{C}$, held at $10^{\circ} \mathrm{C}$. The Taqplus DNA polymerase (Tiangen, China) was used for fidelity. The products from RT-PCR of cDNA and PCR of gDNA were cloned into the pMD18-T (TaKaRa, China), and nine clones were sequenced. The PCR amplification of gDNA was performed with $\mathrm{PF}$ and RP in G. hirsutum and $G$. arboreum, respectively, and the products were cloned and sequenced.

Bioinformatics analysis The DNA sequences were analyzed with DNAstar software and the BLAST program (http://ncbi.nlm.nih.gov). Conserved domain, PROSITE motifs and CAM (calmodulin) binding site were analyzed with NCBI cds Blast (http://www.ncbi.nlm.nih.gov/ structure/), ProDom (http://motif.genome.jp/motif-bin/) and CTDB (http://calcium.uhnres.utoronto.ca), respectively. The ADF protein sequences of different species used for the construction of a phylogenetic tree were downloaded from GenBank. Homological comparison was made using ClustalW software. The phylogenic tree was constructed with Treeview software and the DRAWGRAM program in PHYLIP (http://evolution.genetics.wshington. edu/phylip.html).

Gene expression analysis First-strand cDNA was synthesized from $5 \mu \mathrm{g}$ of total RNA using the StrataScript Kit (Stratagen, USA). The congruent first strand cDNA was used for PCR amplification of GbADF1 ORF with genespecific primers, $\mathrm{PR}$ and $\mathrm{PF}$. The parameters were: $95^{\circ} \mathrm{C}$ for $1 \mathrm{~min}$, followed by 23 cycles of $94^{\circ} \mathrm{C}$ for $1 \mathrm{~min}, 58^{\circ} \mathrm{C}$ 
for $1 \mathrm{~min}, 72^{\circ} \mathrm{C}$ for $1.5 \mathrm{~min}$, and $72^{\circ} \mathrm{C}$ for $10 \mathrm{~min}$. Moreover, the primers 5'-CTGAATCTTCGCTTTCACGTTATC3' and 5'-GGGATGCAAATCTTCGTGAAAAC-3' were used to amplify the ubiquitin cDNA fragments using parameters as $95^{\circ} \mathrm{C}$ for $1 \mathrm{~min}$, followed by 19 cycles of $94^{\circ} \mathrm{C}$ for 1 min, $58^{\circ} \mathrm{C}$ for $1 \mathrm{~min}, 72^{\circ} \mathrm{C}$ for $1.5 \mathrm{~min}$, and $72^{\circ} \mathrm{C}$ for $10 \mathrm{~min}$ (Zhang et al., 2007). The RT-PCR reactions were repeated three times. The products were then separated on a $1.0 \%(\mathrm{w} / \mathrm{v})$ agarose gel.

Expression of the recombinant protein For expression of the $G b A D F 1$, the coding region was inserted into the HindIII and EcoRI sites of vector pET-41a(+) (Novagen, Germany) by intermediate plasmid pMDGbADF1. The expression of the recombinant protein in $E$. coli BL21 (DE3) with the induction of $1 \mathrm{mM}$ IPTG and $2 \%$ glucose after $4 \mathrm{hr}$ at $28^{\circ} \mathrm{C}$ by SDS-PAGE electrophorsis and stained with Coomassie Brilliant Blue R-250.
Western blotting Protein samples, including $25 \mu \mathrm{g}$ of total induced protein with negative control $E$. coli cell harboring no any plasmid, $E$. coli cell harboring pET$41 \mathrm{a}(+)$ and $E$. coli cell harboring pET-GbADF1, were separated by SDS-PAGE, and then visualized by Coomassie blue staining. The protein gel was transferred to a PVDF membrane (Millipore) by using a Trans-Blot SD semi-dry electrophoretic transfer cell (BIO-RAD) for immunoblotting, with $1-2 \mathrm{~mA} / \mathrm{cm}^{2}$ invariable electric current for $1 \mathrm{hr}$. Membrane was blocked with western blot blocking buffer (Tiangen, China) for $1 \mathrm{hr}$ at room temperature with constant agitation. The Anti-His Antibody (Tiangen, China) was diluted with 1:10000 in fresh blocking buffer. Then the membrane was incubated in the primary antibody solution for $1 \mathrm{hr}$ at room temperature with constant agitation. Excess antibody was removed by washing in PBS containing $0.1 \%$ Tween- 20 . The horseradish peroxidase-conjugated anti-rat IgG antibody

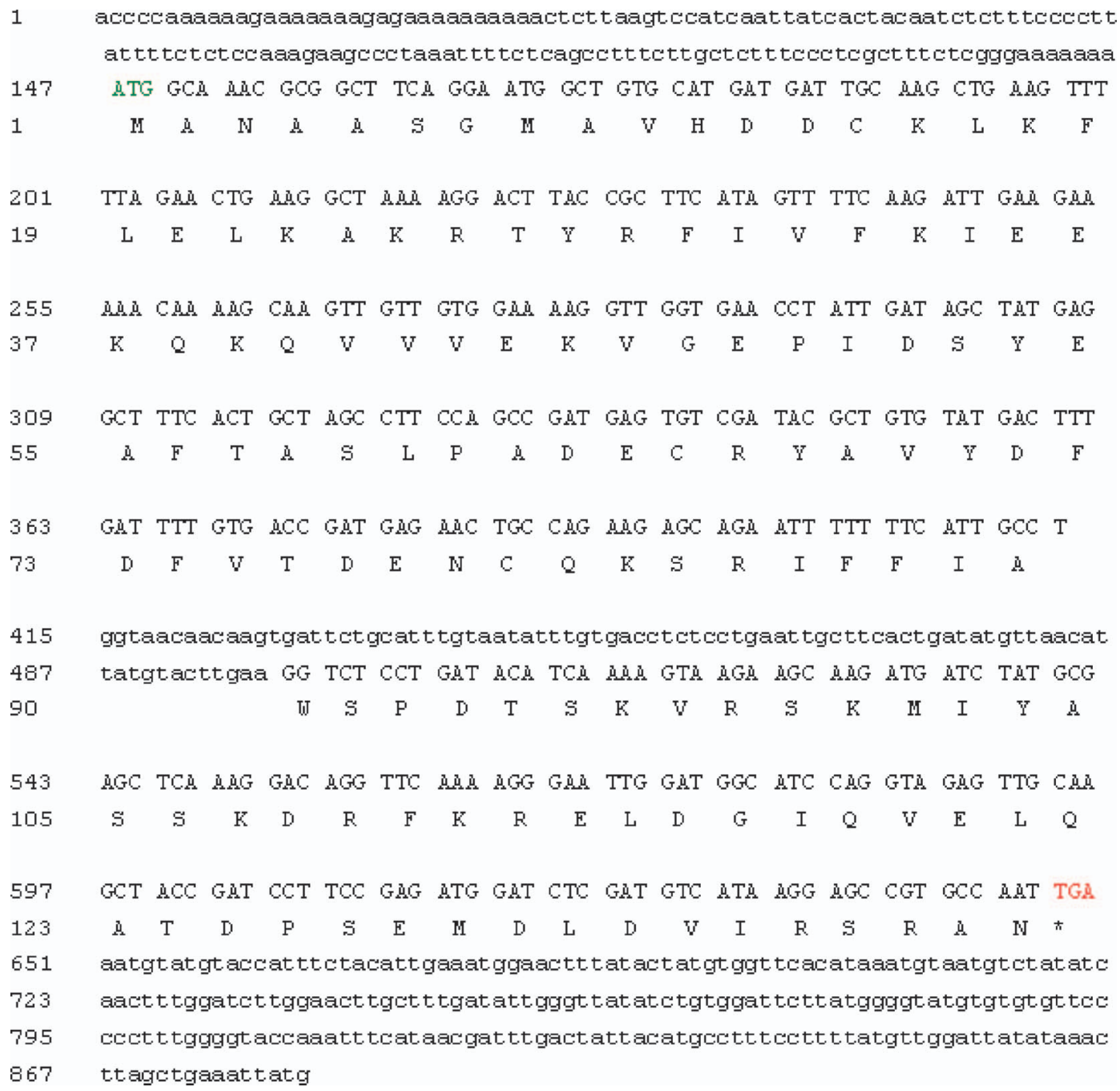

Fig. 1. Nucleotide sequence of $G b A D F 1$ from gDNA and its deduced amino acid sequence. The small letters were untranslated sequence, and the capital letters were coding sequence. The green and red letters indicated initiation and termination codons, respectively. 
(Tiangen, China) was diluted with 1:4000 in fresh blocking buffer for 1-2 $\mathrm{hr}$ at room temperature with constant agitation. After washing the excess antibody, proteins were detected with an enhanced HRP-DAB chromogenic substrate kit (Tiangen, China). Experiments were repeated at least twice using independently prepared samples.

\section{RESULTS}

Sequence characterization and gene structure of GbADF1 An 881 bp gDNA of $G b A D F 1$ was obtained from $G$. barbadense. The nucleotide sequence and deduced amino-acid sequence of this gene were shown in Fig. 1. A 420 bp ORF encoded a protein of 139 amino acids, including $39.57 \%$ nonpolar amino acids, $17.27 \%$ acidic amino acids, $15.83 \%$ basic amino acids and $31.92 \%$ hydrophobic amino aids. The pI was 5.04. A 504 bp fragment was obtained with gDNA by using gene-specific primers. A comparison of the $504 \mathrm{bp}$ fragment and the ORF indicated that the GbADF1 contained an $84 \mathrm{bp}$ intron near the 3 ' end from $287 \mathrm{bp}$ to $371 \mathrm{bp}$.

The 420 bp ORF was also obtained from two cotton species, G. hirsutum and G. arboreum. But there was no intron in the ORF from gDNA of $G$. hirsutum and $G$. arboreum. Therefore, the gene structure of $G b A D F 1$ differed from those of the two cotton species and the other reported $A D F s$ in higher plant (Fig. 2).

High homology of GbADF1 The possible conserved domains were shown in Fig. 3, which indicated that the deduced amino acid sequence included a phosphorylation site, 6-Ser, and a PIP2 binding site. Analysis of CAM potential binding sites indicated that possible CAM combining region was at the $\mathrm{N}$ terminus. There were five homogeneous amino acids substitutions (Leu46/Ile/Val/ Thr, Ile50/Thr/Gly/Lys/Glu/Asn, Asp77/Ala/Ser/Val/Lys/ Asn/Thr, Asp130/Gly/Ser and Ile134/Phe/Val/Leu) in the 20 ADFs of higher plant, which might effect on their structures and functions. In fact, there were only three homogeneous replacements (Asp93/Ser/Thr, Thr94/Val/ Ala and Ala99/Ser) in the PIP2/actin binding site in ADFs of cotton. Different ADFs from higher plant had $76.6 \%$ sequence homology (data not shown). GbADF1 was highly homologous to AtADF1 with 87.8\% amino acids identity and shared the highest homology (99\% identity) with GhADF1, and only one amino acid difference, Ile50/ Phe. ADFs in cotton had high homology from $56 \%$ to
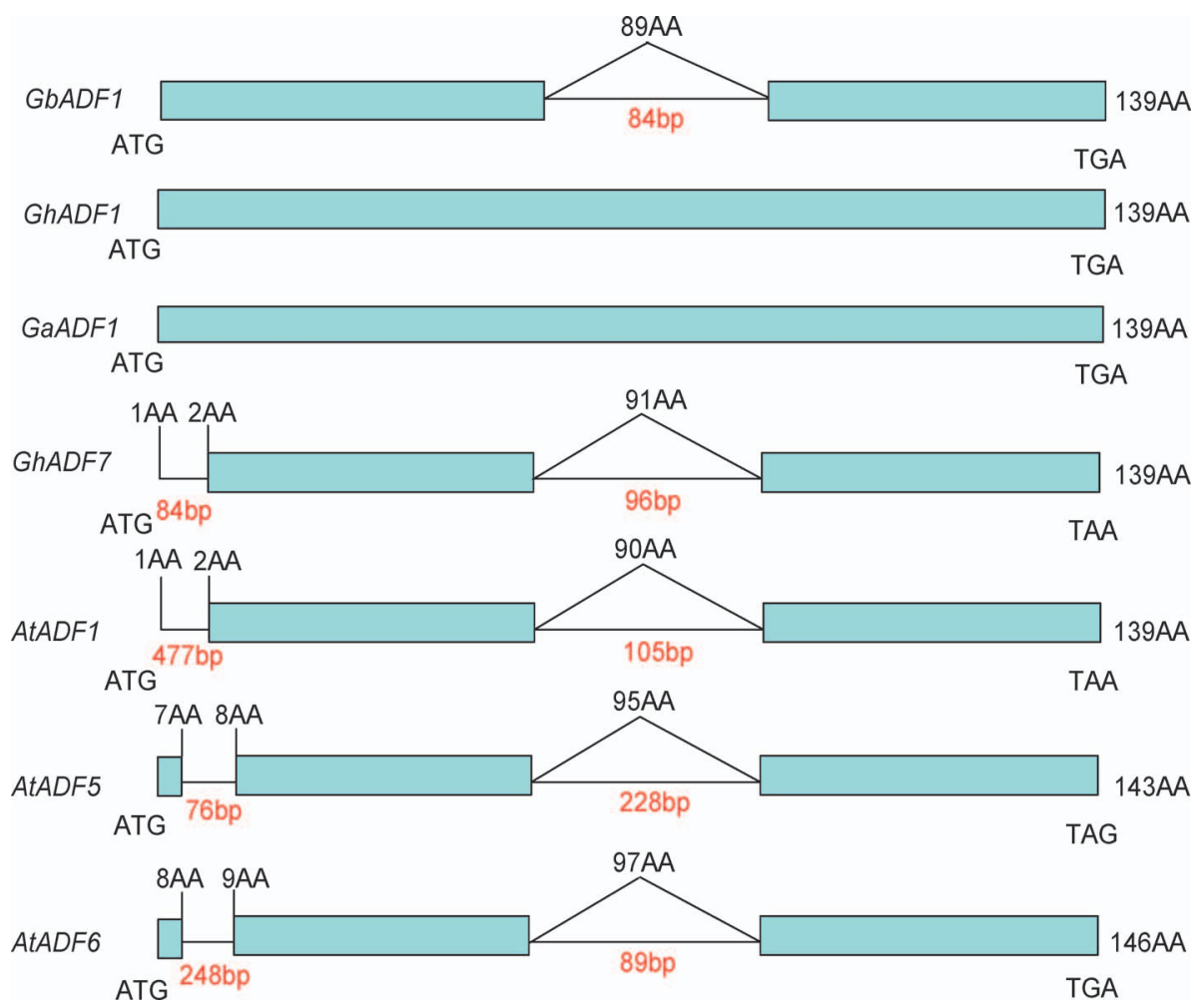

Fig. 2. Position and size of introns in $A D F$ genes. GbADF1, GhADF1 and GaADF1 were described in this study. The sequence of the GhADF7 was described before (Zhang et al., 2006). The sequences of the AtADF1, AtADF5, AtADF6 encoded Arabidopsis ADF (Dong et al., 2001b). The blue box indicated the exon, and the black line indicated the intron. The number of amino acid on the box meant the intron cleavage site. The red number under the black line meant the size of intron. The ATG meant the initiation codon and the TAA, TGA, TAG meant the termination codon. The total number of amino acids of each ORF was in the right of the box. 


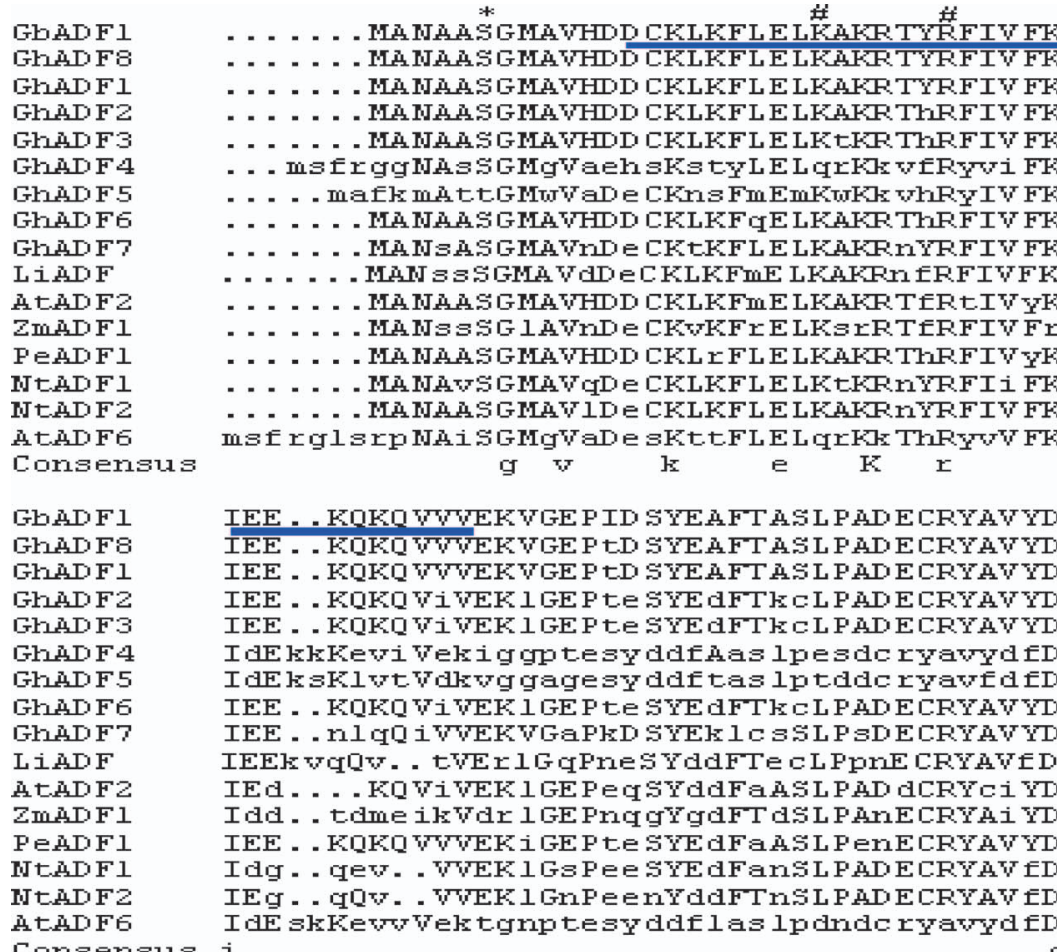

$3: 3$

33

$3: 3$

$3: 3$

$3:$

37

35

$3: 3$

33

33

33

33

$3: 3$

$3=$

GhADF1 FDFVTDEMC QK̈SRI FFIATS PDTSKVRSKMIY A SSKDRFK

GhADF

GhבhDF1

Ghम्मF2

GrhD F3

GhमेD F4

Ghב̈DF 5

GHADF6

GhHDF?

LIADF

A $T A D F 2$

ZMADFl

PEADF 1

WtATF 1

NTADF2

ÄTADFG

consensus

GbADF1

GhAFG

GhमD F1

GHम D F

GHDDF

Grम̈DF4

GHमPD 5

GHम FE

GhADF 7

LiADF

At $\mathrm{ADF} 2$

Zm鸟D 1

PEA $\mathrm{A} D \mathrm{~F}$

WtADF 1

Hof $F 2$

孚形D $F$

Congerams

FD FVTDEWC QKSRI FFIATS PDTSKWRSKM I A A SKDRFK FD FVTDEWC DKSRI FFIATS FDTSKWRSKM I FDF 1 TaEW DKSRI FFIATS PDTSx I FSKMI Y ASKDR FK FDF 1TaEWvPKSRI FFIATS FDTSェ FSKMI I ASFDR FK

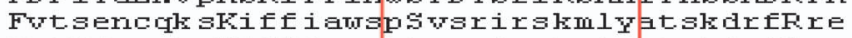
FvtVdror sKiffiawsot asrirakmlyat FDF $1 T$ TEWvOKSRI FFI

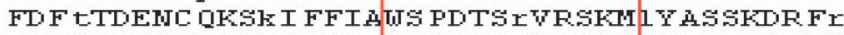
FDFWTDENCQRSK IFF I 3 WS DTS TWR SFM.TA S TFDRFF FD FVTaEHC QKSK I FFIATS FDT AKV RdKMIT A SSKDR FK

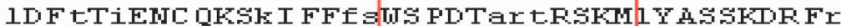
FD FVTaEWC QKSRI FFIAWC PDTATVRSKM I Y ASSKDR FK

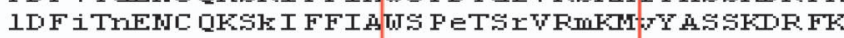
FDFITEENC QKSkI FFIAWS FDTSKWFMKM $\rightarrow$ A ASFDR FF

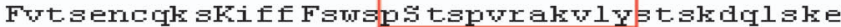
$\mathrm{k}$

RELDGIOVE LQATD F'SEMDL DVIRS̈RAN RE L DGIQVE LQATD F SEMD L DVIRSRAN RE L DGIDVE LDATD F'SENDL DVIRSR AN REL DGIQVE LQÄTD F' TEMGL DVIE SR.Ä RELDGIOVE LOATD F TEMGLDVIK SR AN lEgihYeiqatdptemdlevireRah ldgihyevqatdpt emgMIVikhk:ay REL DGIQVE L DATD P TEMgL DVEK SRAW RE L DGVOVE L QATD F SEM RELD GI DWEL DATDPSEM $3 \mathrm{mD}$ i IK FE L DGIQVE L QATD F tEMGL DVEK SR tW REL DGIDOE I DATD P SEM L D I VRSR TW RE L DGI DVE L DACD F tEMGL DVI GSRA RELDGIDVE L DATD F SEM RE L DGIDVE L DATD P SEM lqgihyeiqatdptevilev lrekan

Fig. 3. Amino acid sequence alignment of the GbADF1. The asterisk indicated the position of the putative phosphorylation site at the N-terminus. The well symbol indicated the position of the putative nuclear binding site. The point indicated the position of the putative special F-actin bindng site. The red rectangle and blue line showed the PIP2/actin binding site and the possible CAM combining region, respectively. Accession numbers: GhADF1 (AAY88048), GhADF2 (ABD63906), GhADF3 (ABD66505), GhADF4 (ABD66506), GhADF5 (ABD66507), GhADF6 (ABD66508), GhADF7 (ABD66503), GhADF8 (ABD66504), AtADF1 (NP_190187), AtADF2 (NP_566882), AtADF3 (NP_851227), AtADF4 (NP_851228), AtADF5 (NP_565390), AtADF6 (AAM63510), PeADF1 (AAK72617), PeADF2 (AAK72616), NtADF1 (AAL91666), NtADF2 (AAL91667), ZmADF1 (P46251). 
$100 \%$ (data not shown).

To study the phylogeny of the ADFs, the complete protein sequences were aligned, and the phylogenetic tree was generated by the neighbor-joining method using 25 ADFs including GbADF1 (Fig. 4). The tree indicated that the $25 \mathrm{ADFs}$ in plants, including all 11 Arabidopsis $\mathrm{ADFs}$, subclass-representative rice (Oryza sativa) and all 9 Gossypium ADFs. They were assembled using clustralW, and the protein destrin from human (DEST1_human, replaced by DEST_Pig in 2005) was a outgroup. The plant ADF proteins were grouped into four subclasses according to the output tree. The dicot cotton and the monocot rice were ranged in each subclass. The data suggested that the three plant spe- cies had evolved with a large pre-existing repertoire of ADFs. The GbADF1 and AtADF1,-2,-3,-4 were clustered in the subclass I, which expressed constitutively in all vegetative and reproductive tissues except pollen (Ruzicka et al., 2007).

Expression profiles of $G B A D F 1$ in different fiber developmental stage and multi-tissues To determine the expression pattern of $G b A D F 1$, semi-quantitative RT-PCR analysis was performed. The results showed that all tissues contained GbADF1 mRNA. Higher level was detected in fiber tissues than in trophic tissues (Fig. 5). This revealed that $G b A D F 1$ was a constitutive expression gene in cotton, and the expression levels might

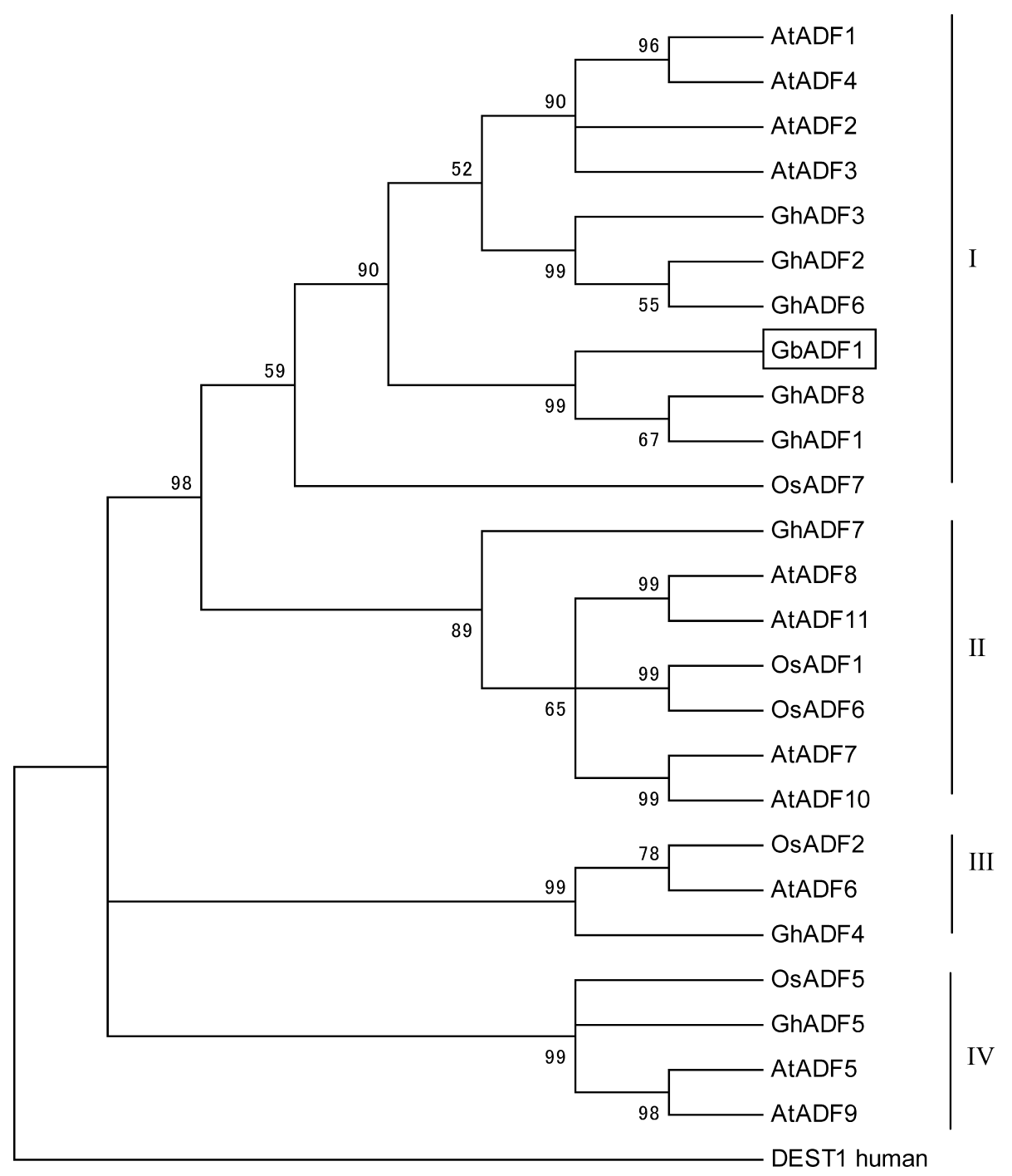

Fig. 4. ADF phylogeny. Neighbor-joining protein sequence phylogeny showed all 11 AtADFs, all nine ADF proteins from Gossypium and representatives from Oryza. Some accession numbers were the same as Fig. 3, and other accession numbers were as follows: AtADF7(AAQ65136), AtADF8(NP_567182), AtADF9(NP_195223), AtADF10(NP_568769), AtADF11(NP_171680), OsADF1(Q6EUH7), OsADF2(Q9AY76), OsADF5(Q10P87), OsADF6(Q7SXN9), OsADF7(Q0DLA3), DEST1_human(P60982). The Arab numbers meant the bootstrap values. Subclasses I to IV were indicated by vertical bars on the right. 


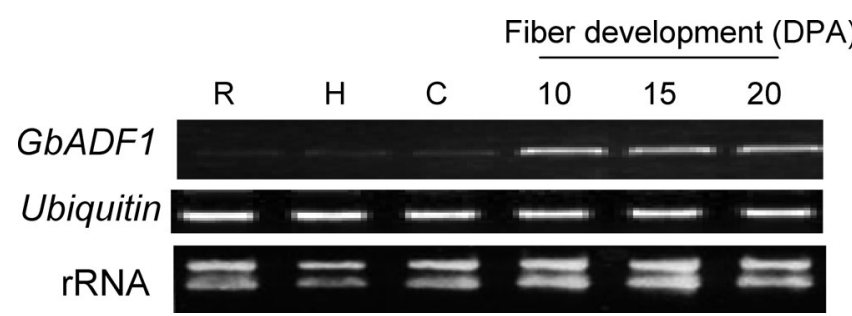

Fig. 5. Semi-quantitative RT-PCR analysis of GbADF1 expressed in different tissues and fiber developing stages. 20: DPA20, 15: DPA15, 10: DPA10, R: root, H: hypocotyls, C: cotyledon. Ubiquitin: the internal control for RT-PCR.

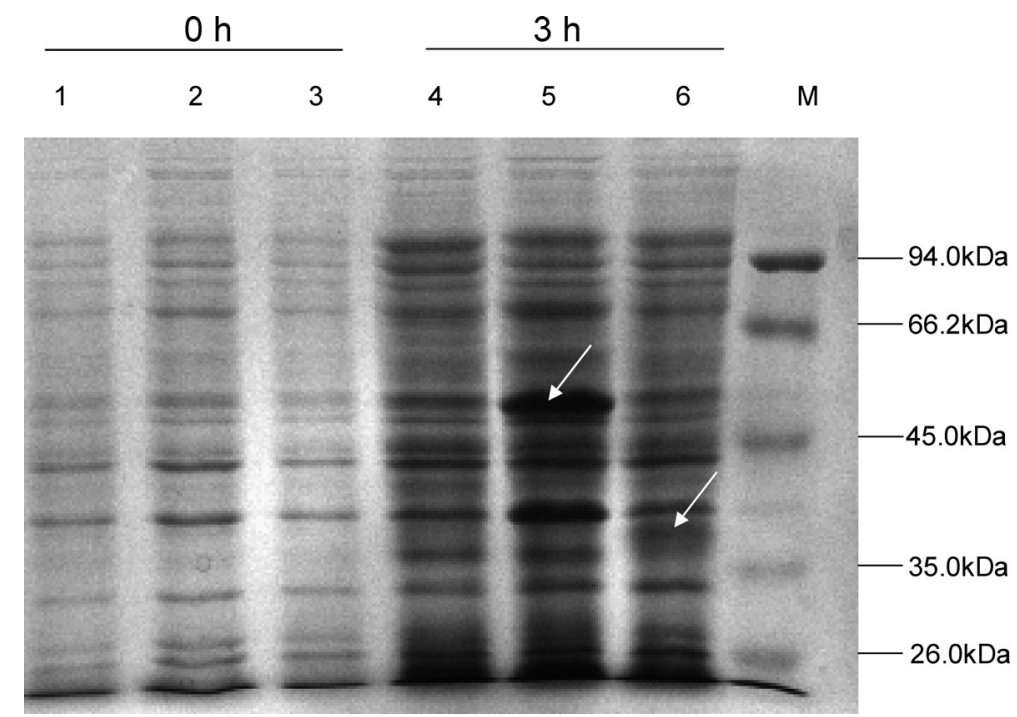

Fig. 6. Expression of the recombinant GbADF1 in E. coli. M: ladder marker; 1 and 4: control $E$. coli cell harboring no plasmid; 2 and 5: $E$. coli cell harboring pET-GbADF1. The white arrow indicated the position of the GbADF1 recombinant protein. 3 and 6: $E$. coli cell harboring pET-41a $(+)$. The white arrow indicated the position of the pET-41 tag protein. Proteins were separated by SDS-PAGE and stained with Coomassie Brilliant Blue. $0 \mathrm{hr}$ meant the non-induced cell. $4 \mathrm{hr}$ meant that the cell was produced with IPTG for $4 \mathrm{hr}$.

vary in trophic tissues and fiber tissues.

Prokaryotic expression the GbADF1 protein So far, no cotton ADFs was expressed in E. coli. Compared with the control of non plasmid $E$. coli cell and harboring pET$41 \mathrm{a}(+)$, the $E$. coli cell harboring pET-GbADF1 could be induced by IPTG, and the recombinant His-tagged and GST-tagged GbADF1 was expressed (Fig. 6). The recombinant GbADF1 was approximately $50 \mathrm{kDa}$. The protein before the multiple cloning sites, including His and GST was about $35.6 \mathrm{kDa}$. Thus, GbADF1 was a $\sim 15 \mathrm{kDa}$ peptide.

Western blotting analysis No bands were discovered in the negative control $E$. coli cell harboring no plasmid by using western blotting. About $36 \mathrm{kDa}$ protein from $E$. coli cell harboring pET-41a(+) expressed tag had been tested by His-tag antibody. The recombinant protein of about $50 \mathrm{kDa}$ was present at substantial levels in $E$. coli cell harboring pET-GbADF1 (Fig. 7). These data con-

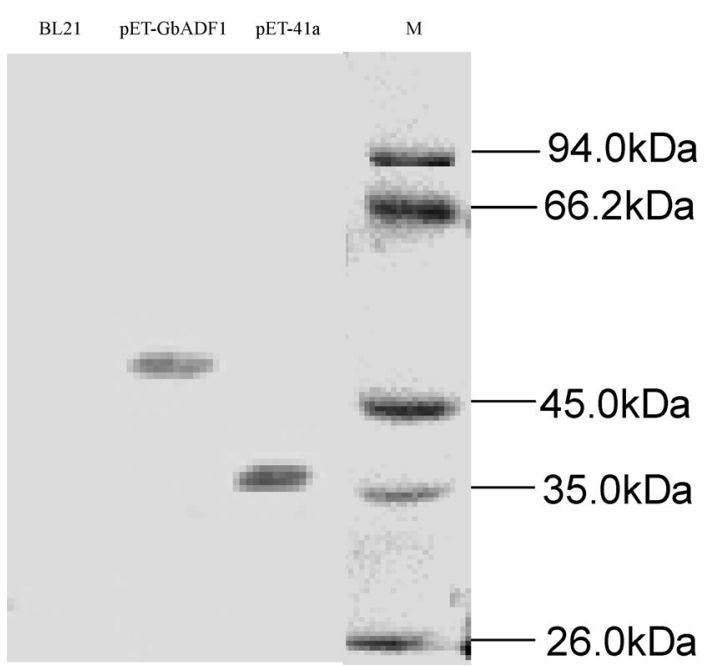

Fig. 7. Western blotting analysis of $G b A D F 1$ expression in $E$. coli. BL21: negative control $E$. coli cell harboring no plasmid; pET-GbADF1: $E$. coli cell harboring pET-GbADF1; pET41a(+): E. coli cell harboring pET-41a(+); M: protein molecular marker. 
firmed the existence of a protein corresponding to GbADF1.

\section{DISCUSSION}

In this study, GbADF1 with a 420 bp ORF was cloned and characterized. The gene encoded a protein of 139 amino acids with two conserved domains, 6-Ser and the PIP2/actin binding site. Its amino acid sequence was similar to that of the $\mathrm{ADF} /$ cofilin family in other plants. The gene structure of GbADF1 differed from that of the other reported $A D F s$ in higher plant. An $84 \mathrm{bp}$ intron existed at the beginning of the PIP2/actin binding site from $287 \mathrm{bp}$ to $371 \mathrm{bp}$ in gDNA. Semi-quantitative RTPCR showed higher levels $G b A D F 1$ in fibers than in trophic tissues, suggesting that it might be involved in regulating the development of cotton fiber.

None of cotton ADFs was expressed in $E$. coli in previous researches. In this study, the results from western blotting and the expression in prokaryote confirmed the existence of a protein corresponding to GbADF1, and the GbADF1 protein would be $\sim 15 \mathrm{kDa}$. The real molecular weight of GbADF1 was just in the range of theoretically deduced molecular weight, $15-22 \mathrm{kDa}$, in $\mathrm{ADF}$ family (Staiger et al., 1997).

The structure of GhADF7 showed two introns existed as found in the most AtADFs (Zhang et al., 2006). But no more structure reports of $G h A D F s$ were found. In this study, the ORFs of ADF were cloned from other two cotton species, G. hirsutum and G. arboreum, respectively. There were no introns in the ADFs of the two cotton species. As far as can currently be determined, plant $\mathrm{ADF} /$ cofilin genes are organized in a similar manner, with an intron following the exon encoding the amino terminus and a conserved intron further 3'. This pattern held for Arabidopsis and Oryza sativa ADF/cofilin genes (Maciver and Hussey, 2002). The structure of GbADF1 was different from this manner. GbADF1 had only one intron near the 3 ' end just before the $\alpha$-helix 3 which had been researched in comparative structural analysis of the ADF/ cofilin family (Bowman et al., 2000). And the intron of GbADF1 was at the beginning of the PIP2/actin binding site, which indicated that there were some differences on the ontogeny and evolution.

As we know, actin is a major, dynamic component of the plant cytoskeleton. The plant actin gene family could be divided into two major classes, a vegetative class and a reproductive class (Mun et al., 2000). A clear specialization between vegetative and reproductive isovarians had also been observed in profilin (Staiger et al., 1997). The thorough analysis of the ADF gene family in Arabidopsis was done by tissue-specific expression patterns and subcellular localization (Ruzicka et al., 2007). The phylogenic tree indicated that the two ancestral ADF genes, vegetative-expressed and pollen-expressed genes, had acquired their tissue specificity before monocot-dicot divergence, which was also supported by this research. Gossypium, Arabidopsis and Oryza sativa evolved with a large pre-existing repertoire of ADFs. And the data also indicated that the ADFs had important functions in flowering plants. Just as the phylogenic tree described, GbADF1 was constitutive expression gene in cotton, and the GbADF1 belonged to subclass I, which was classified into the predominant vegetative group of $\mathrm{ADF}$ isovariants in the plant in the four subclasses (Ruzicka et al., 2007). The promoter analysis would be done to discuss expression profile of GbADF1. Otherwise, $\mathrm{ADF}$ was more potent in depolymerization than cofilin (Giuliano et al., 1988). The function in cotton fiber development would require more detailed research.

This research was supported by the National 973 Project (No. 2004CB117302), the National 863 Project (No. 2006011001044), the National Transgenic Breeding Project (2008ZX08009-003), the Natural Science Foundation of China (No. 30671322, 30871561) and the Natural Science Foundation of Hebei Province (No. C2006001034).

\section{REFERENCES}

Aizawa, H., Sutoh, K., Tsubuki, S., Kawashima, S., Ishii, A., and Yahara, I. (1995) Identification, characterization, and intracellular distribution of cofilin in Dictyostelium discoideum. J. Biol. Chem. 270, 10923-10932.

Bamburg, J. R., McGough, A., and Ono, S. (1999) Putting a new twist on actin: ADF/cofilins modulate actin dynamics. Trends Cell Biol. 9, 364-370.

Bowman, G. D., Nodelman, I. M., Hong, Y., Chua, N. H., Lindberg, U., and Schutt, C. E. (2000) A comparative structural analysis of the ADF/cofilin family. Proteins 41, 374384 .

Dong, C. H., Xia, G. X., Hong, Y., Ramachandran, S., Kost, B., and Chua, N. H. (2001a) ADF proteins are involved in the control of flowering and regulate F-actin organization, cell expansion, and organ growth in Arabidopsis. Plant Cell 13, 1333-1346.

Dong, C. H., Kost, B., Xia, G. X., and Chua, N. H. (2001b) Molecular identification and characterization of the Arabidopsis AtADF1, AtADF5, AtADF6 genes. Plant Mol. Biol. 45, 517-527.

Gibbon, B. C. (2001) Actin monomer-binding proteins and the regulation of actin: dynamics in plants. J. Plant Growth Regul. 20, 103-112.

Giuliano, K. A., Khatib, F. A., Hayden, S. M., Daoud, E. W., Adams, M. E., Amorese, D. A., Bernstein, B. W., and Bamburg, J. R. (1988) Properties of purified actin depolymerizing factor from chick brain. Biochemistry 27, 89318938.

Gunsalus, K. C., Bonaccorsi, S., Williams, E., Verni, F., Gatti, M., and Goldberg, M. L. (1995) Mutations in twinstar, a Drosophila gene encoding a cofilin / $A D F$ homologue, result in defects in centrosome migration and cytokinesis. J. Cell Biol. 131, 1243-1259.

Hussey, P. J., Yuan, M., Calder, G., Khan, S. and Lioyd, C. W. (1998) Microinjection of pollen-specific actin-depolymerizing factor, ZmADF1, reorients F-actin strands in Tradescantia stamen hair cells. Plant J. 14, 353-357. 
Hussey, P. J., Ketelaar, T., and Deeks, M. J. (2006) Control of the actin cytoskeleton in plant cell growth. Annu. Rev. Plant Biol. 57, 109-125.

Iida, K., Moriyama, K., Matsumoto, S., Kawasaki, H., Nishida, E., and Yahara, I. (1993) Isolation of a yeast essential gene, COF1, that encodes a homologue of mammalian cofilin, a low-Mr actin-binding and depolymerizing protein. Gene 124, 115-120.

Kim, S. R., Kim, Y. and An, G. (1993) Molecular cloning and characterization of anther-preferential cDNA encoding a putative actin-depolymerizing factor. Plant Mol. Biol. 21, 39-45.

Lappalainen, P., and Drubin, D. G. (1997) Cofilin promotes rapid actin filament turnover in vivo. Nature 388, 78-82.

Lopez, I., Anthony, R. G., Maciver, S. K., Jiang, C. J., Khan, S., Weeds, A. G., and Hussey, P. J. (1996). Pollen specific expression of maize genes encoding actin depolymerizing factor-like proteins. Proc. Natl. Acad. Sci. USA 93, 74157420 .

Maciver, S. K., and Hussey, P. J. (2002) The ADF/cofilin family: actin-remodeling proteins. Genome Biol. 3, 3007.1-3007. 12.

Moon, A. L., Janmey, P. A., Louie, K. A., and Drubin, D. G. (1993) Cofilin is an essential component of the yeast cortical cytoskeleton. J. Cell Biol. 120, 421-435.

Mun, J. H., Yu, H. J., Lee, H. S., Kwon, Y. M., Lee, J. S., Lee, I., and Kim, S. G. (2000) Two closely related cDNA encoding actin-depolymerizing factors of Petunia are mainly expressed in vegetative tissues. Gene 257, 167-176.
Paterson, A., Brubaker, C., and Wendel, J. A. (1993) A rapid method for extraction of Gossypium spp. genomic DNA suitable for RFLP or PCR analysis. Plant Biol. Reporter 11, 122-127.

Peng, S. Q., and Huang, D. F. (2006) Expression of an Arabidopsis actin-depolymerizing factor 4 gene $(A t A D F 4)$ in tobacco causes morphological change of plants. J. Plant Physiol. Molecular Biol. (in Chinese) 32, 52-56.

Rozycka, M., Khan, S., Lopez, I., Greenland, A. J., and Hussey, P. J. (1995) A Zea mays pollen cDNA encoding a putative actin depolymerizing factor. Plant Physiol. 107, 10111012.

Ruzicka, D. R., Kandasamy, M. K., McKinney, E. C., BurgosRivera, B., and Meagher R. B. (2007) The ancient subclasses of Arabidopsis ACTIN DEPOLYMERIZING FACTOR genes exhibit novel and differential expression. Plant J., 52, 460-472.

Staiger, C. J., Gibbon, B. C., Kovar, D. R., and Zonia, L. E. (1997) Profilin and actin-depolymerizing factor: modulators of actin organization in plants. Trends Plant Sci. 2, 275-281.

Zhang, C. W., Huang, G. Q., Xu, W. L., Wang, X. L., and Li, X. B. (2006) Analysis of cotton GhADF7 gene structure and evolutionary relationship. Journal of Central China Normal University (Nat. Sci.) 40, 575-579.

Zhang, C. W., Guo, L. L., Wang, X. L., Zhang, H., Shi, H. Y., Xu, W. L., and Li, X. B. (2007) Molecular characterization of four $A D F$ genes differentially expressed in cotton. J. of Genetics and Genomics 34, 347-354. 